\title{
Efficacy of Combination Therapy with Linalool and Doxorubicin Encapsulated by Liposomes as a Two-in-One Hybrid Carrier System for Epithelial Ovarian Carcinoma
}

This article was published in the following Dove Press journal:

International Journal of Nanomedicine

\author{
Tae $\ln \mathrm{Wi}^{1, *}$ \\ Ji Eun Won ${ }^{1, *}$ \\ Chan Mi Lee' \\ Jeong-Won Lee ${ }^{2}{ }^{2}$ \\ Tae Heung Kang' \\ Byung Cheol Shin ${ }^{3}$ \\ Hee Dong Han (I) \\ Yeong-Min Park' \\ 'Department of Immunology, School of \\ Medicine, Konkuk University, Chungju \\ 380-70I, South Korea; ${ }^{2}$ Department of \\ Obstetrics and Gynecology, Samsung \\ Medical Center, Sungkyunkwan \\ University School of Medicine, Seoul, \\ South Korea; ${ }^{3} \mathrm{Bio} /$ Drug Discovery \\ Division, Korea Research Institute of \\ Chemical Technology, Daejeon, South \\ Korea
}

*These authors contributed equally to this work
Correspondence: Hee Dong Han; Yeong-Min Park

Department of Immunology, School of Medicine, Konkuk University, 268

Chungwondae-Ro, Chungju,

Chungcheongbuk-Do 380-70I, South

Korea

Tel +82-2-2030-7848; +82-2-2049-6330

Fax +82-2-2049-6192

Email hanhd@kku.ac.kr;

immun3023@kku.ac.kr
Background: Epithelial ovarian cancer (EOC) is a fatal gynecologic malignancy that is usually treated with chemotherapy after surgery. However, patients who receive chemotherapy experience severe side effects because of the inherent toxicity and high dose of chemotherapeutics. To overcome these issues, we suggest a combination therapeutic strategy using liposomes encapsulating linalool nanoemulsions (LN-NEs) and doxorubicin (DOX), a chemotherapeutic drug, to increase their synergistic antitumor efficacy and reduce the incidence of side effects from chemotherapeutics for EOC.

Methods: The physical properties of LN-NE-DOX-liposomes were characterized by light scattering with a particle size analyzer. Cell viability was determined by MTT assay. Therapeutic efficacy was evaluated in a mouse HeyA8 EOC tumor model of ovarian carcinoma. Additionally, biochemical toxicity was analyzed for levels of aspartate aminotransferase (AST), alanine aminotransferase (ALT), and blood urea nitrogen (BUN) using $\mathrm{BALB} / \mathrm{c}$ nude mice.

Results: The size of the liposomes encapsulating LN-NEs and DOX (LN-NE-DOXliposomes) was $267.0 \pm 4.6 \mathrm{~nm}$, with a loading efficiency of $55.1 \pm 3.1 \%$ and $27.2 \pm 0.9 \%$ for linalool and DOX, respectively. Cell viability after treatment with LN-NE-DOXliposomes was significantly decreased compared to that of cells treated with DOX liposomes, and apoptosis was significantly increased. Additionally, LN-NE-DOX-liposomes significantly inhibited HeyA8 EOC tumor growth compared to that of the control $(p<0.01)$ and DOX-liposome-treated groups $(p<0.05)$, while decreasing cell proliferation (Ki67) and microvessel density (CD31), and promoting apoptosis (caspase-3) compared to the control $(p<0.05)$. Moreover, the liposomal formulations induced no significant differences in biochemical toxicity (AST, ALT, and BUN) compared to healthy control mice, indicating that the liposomal formulations showed no overt toxicity in mice.

Conclusion: This study demonstrates that the production of LN-NE-DOX-liposomes is a pivotal approach for EOC treatment, suggesting a novel combination therapeutic strategy. Keywords: linalool, liposome, ovarian carcinoma, doxorubicin, combination therapy

\section{Introduction}

Epithelial ovarian cancer (EOC) is a common gynecologic malignancy and a major cause of cancer-related deaths, with more than 200,000 new cases and 150,000 deaths worldwide annually. ${ }^{1}$ Ovarian cancer originates from the epithelial, stromal, and germ cells, and EOC makes up more than $90 \%$ of all ovarian cancer cases. ${ }^{1}$ 
Generally, patients with EOC receive paclitaxel and platinum-based chemotherapy (cisplatin) in a cocktail form after primary surgery, but a number of patients experience side effects after chemotherapy. ${ }^{2}$ After primary chemotherapy fails in patients with EOC, doxorubicin (DOX) is used as a secondary chemotherapy. However, in secondary chemotherapy, EOC patients still feel side effects due to DOX. Therefore, an effective strategy that would reduce the incidence of these side effects and increase therapeutic efficacy is needed.

Therapeutic strategies, including chemotherapy, ${ }^{3}$ immunotherapy, ${ }^{4}$ and radiotherapy ${ }^{5}$ have recently been developed for the treatment of EOC. Although chemotherapy is still commonly used as a treatment method, the side effects of anticancer drugs associated with toxicity in normal cells and organs have caused severe problems such as nausea, vomiting, and cardiotoxicity. ${ }^{6,7}$ To overcome these concerns, effective carrier systems for the delivery of chemotherapeutics are being actively developed to increase delivery efficiency in the tumor microenvironment. Among these systems, liposomes are highly attractive carriers that not only transport anticancer drugs safely to tumor cells but they also increase blood circulation time, thereby enhancing drug delivery efficiency in the tumor microenvironment. ${ }^{8}$ Moreover, the unique properties of liposomes, such as their ability to encapsulate various drugs and their tunable surface modification, offer many advantages over their counterparts. Notably, several liposome formulation-based therapeutic nanomedicine agents have already been approved by the Food and Drug Administration (FDA) for clinical use, and the potential use of natural compounds as anticancer drugs has also been vigorously studied. ${ }^{9-12}$ Among anticancer drugs, we used DOX because of its hydrophilic characteristics, making it attractive for the development of liposomal formulations. Here, we utilized a remote loading method, which could load DOX into liposomes through the driving force of a transmembrane ammonium gradient. ${ }^{13}$ Linalool (LN), a natural compound, exhibits antitumor effects by increasing the production of reactive oxygen species (ROS), promoting cell apoptosis due to oxidative stress. ${ }^{14} \mathrm{LN}$ is composed of a monoterpene tertiary alcohol and can be obtained from certain plants as an essential oil. ${ }^{15}$ However, LN is highly insoluble in water due to the nonpolar nature of hydrocarbons, leading to low bioavailability, limiting the use of $\mathrm{LN}$ as a chemotherapeutic agent. ${ }^{15,16}$ To overcome this limitation, we developed an $\mathrm{LN}$-incorporated nanoemulsion (LN-NE) system as a therapeutic agent. ${ }^{17}$
To enhance their therapeutic efficacy against EOC, combination strategies have focused on the use of two or more therapeutic agents. ${ }^{18}$ As a novel strategy to eradicate cancer, combination therapies have shown greater therapeutic benefit compared to monotherapies because of the different biological mechanisms of anticancer drugs that can show synergistic effects. ${ }^{19,20}$ Based on this study, we propose a combination strategy using a liposomal platform that incorporates both LN and DOX, which exerted promising synergistic effects for treatment of EOC.

\section{Materials and Methods \\ Materials}

Hydrogenated soy phosphatidylcholine (HSPC), cholesterol (CHOL), and 1,2-distearoyl-sn-glyecero-3-phosphoethanolamine-N-[methoxy(polyethylene glycol)-2000] (DSPEmPEG-2000) were purchased from Avanti Polar Lipids Inc. (Alabaster, AL, USA). Linalool (LN) and doxorubicin hydrochloride (DOX) were purchased from Sigma-Aldrich (St. Louis, MO, USA). RPMI 1640 and fetal bovine serum (FBS) were purchased from Welgene (Gyeonsan, Korea). The other materials were of analytical grade and used without further purification.

\section{Cell Culture Conditions}

The derivation and source of human epithelial ovarian cancer cell lines HeyA8 have been received from Dr. A. K. Sood (MD Anderson Cancer Center, USA, 2014) and previously described. ${ }^{21,22}$ The use of the HeyA8 cells was approved by the committee of Konkuk University (South Korea, Ref. No: KU18200). Cells were maintained in RPMI 1640 medium supplemented with $0.1 \%$ gentamycin (Sigma-Aldrich, St. Louis, MO, USA) and 10\% FBS (Biowest, Nuaille, France). ${ }^{21,23}$ The cells were tested for mycoplasma, hepatitis, and Sendai virus infection at the Korea Research Institute of Bioscience and Biotechnology (Ref. NO. M16442, South Korea). The cells were free of Mycoplasma and inspected at 2016. All in vitro and in vivo experiments were conducted when cells reached $70 \%$ and $80 \%$ confluency at passage 5 .

\section{Preparation of LN-NEs and LN-NE-DOX -Liposomes}

LN-NEs were prepared by the self-nano-emulsifying drug delivery system (SNEDDS) method in a $4 \%$ Tween 80 solution. ${ }^{17}$ Briefly, LN $(0.87 \mathrm{~g} / \mathrm{mL})$ was added to the $4 \%$ Tween 80 solution and stirred for $10 \mathrm{~min}$ at $24{ }^{\circ} \mathrm{C}$. LN-NEs 
were obtained by removing unreacted Tween 80 and LN using a dialysis membrane (Spectrum Labs, Rancho Dominguez, CA, USA) at $4{ }^{\circ} \mathrm{C}$ for $24 \mathrm{~h}$.

DOX-liposomes were prepared by a thin lipid film hydration method. ${ }^{24}$ HSPC, CHOL, and DSPE-mPEG $-2000(3: 1: 1, \mathrm{w} / \mathrm{w})$ were mixed and dissolved in a solution of chloroform and methanol $(8: 2, \mathrm{v} / \mathrm{v})$. The organic solvent was removed using a rotary evaporator (WiseVaporTMEv-1001, SciLab, South Korea) under vacuum at $41{ }^{\circ} \mathrm{C}$ to obtain a thin lipid film. The lipid film was hydrated using an aqueous solution of $250 \mathrm{mM}$ ammonium sulfate. The liposomal solution was sonicated using a probe-type sonicator (20\% amplitude, $5 \mathrm{~s}$ pulses on and $3 \mathrm{~s}$ pulses off, $5 \mathrm{~min}$, VCX 500, Sonics, USA), and free ammonium sulfate was removed using a Sephadex G-50 column. DOX was encapsulated into liposomes using a remote loading method. ${ }^{13}$ Briefly, the DOX solution $(2 \mathrm{mg} / \mathrm{mL})$ was mixed with liposomes and incubated at $60{ }^{\circ} \mathrm{C}$ for $24 \mathrm{~h}^{13}$ Free DOX was removed using a Sephadex G-50 column.

LN-NE-DOX-liposomes were prepared by adding LNNEs in the hydration procedure for the DOX-liposome preparation as described above. The loading efficiency of LN and DOX in the liposomes were measured by a UV-vis spectrophotometer (UV-mini, Shimadzu, Japan) at $295 \mathrm{~nm}$ and $490 \mathrm{~nm}$, respectively. ${ }^{25}$ The loading efficiency of LN was calculated according to the formula: Loading efficiency of $\mathrm{LN}(\%)=\left(\mathrm{F}_{1}-\mathrm{F}_{0} / \mathrm{F}_{\mathrm{tl}}-\mathrm{F}_{0}\right) \times 100$, where $\mathrm{F}_{1}$ is the $\mathrm{LN}$ into LN$\mathrm{NE}$ after dissolution with $70 \%$ ethanol, $\mathrm{F}_{0}$ is background of the $70 \%$ ethanol, and $\mathrm{F}_{\mathrm{tl}}$ is the $\mathrm{LN}$ of initial amount of LN. Additionally, the loading efficiency of DOX was calculated according to the formula: Loading efficiency of DOX $(\%)=$ $\left(\mathrm{F}_{\mathrm{d}}-\mathrm{F}_{0} / \mathrm{F}_{\mathrm{td}}-\mathrm{F}_{0}\right) \times 100$, where $\mathrm{F}_{\mathrm{d}}$ is the DOX of liposome after dissolution with $0.1 \%$ Triton $\mathrm{X}-100, \mathrm{~F}_{0}$ is the background of detergent solution, and $\mathrm{F}_{\mathrm{td}}$ is the DOX of initial DOX. The size and zeta potential of the liposomes were measured by dynamic light scattering using an electrophoretic light scattering photometer (SZ-100, Horiba, Kyoto, Japan). The morphology of the LN-NE-DOX-liposomes was observed by cryogenic transmission electron microscopy (Tecnai T12 Bio-TWIN, FEI Company, Hillsboro, OR, USA). ${ }^{26}$

\section{In vitro Cytotoxicity Assay}

The cytotoxicity of LN-NEs, DOX-liposomes, and LN-NEDOX-liposomes was determined by the MTT assay. ${ }^{17}$ HeyA8 cells were seeded at a density of 3000 cells/well in 96-well plates in triplicate and cultured overnight at $37^{\circ} \mathrm{C}$ in an incubator containing $5 \% \mathrm{CO}_{2}$. After the addition of
LN-NE, DOX-liposomes, or LN-NE-DOX-liposomes in the cell culture medium, the cells were further incubated for $24 \mathrm{~h}, 48 \mathrm{~h}$, and $72 \mathrm{~h}$. Absorbance was measured using a microplate reader (EL808, Bio-Tek, Winooski, VT, USA) at $560 \mathrm{~nm}$.

\section{Apoptosis Assay}

HeyA8 cells were seeded in a 6-well plate at a density of $1 \times$ $10^{5}$ cells/well and cultured overnight at $37{ }^{\circ} \mathrm{C}$ in an incubator containing $5 \% \mathrm{CO}_{2}$. LN-NEs, DOX-liposomes, or LN-NE-DOX-liposomes were added to the cells and further incubated for $72 \mathrm{~h}$ (DOX: $0.5 \mu \mathrm{M}, \mathrm{LN}$ : $0.3 \mathrm{mM}$ ). Cells were then stained with FITC-annexin V (Biolegend, San Diego, CA, USA) and 7-Amino-Actinomycin D (7-AAD) (BD Biosciences, Franklin Lakes, NJ, USA). ${ }^{17}$ Apoptotic cells were analyzed by flow cytometry (BD FACSCalibur with CELLQuest software, BD Biosciences, Franklin Lakes, NJ, USA).

\section{Therapeutic Efficacy of LN-NE-DOX- Liposomes}

Female BALB/c nude mice (5-6 weeks old, 20 g) were purchased from ORIENT (Gapyeong, South Korea). The protocol was approved by the Institutional Animal Care and Use Committee of Konkuk University (South Korea, Ref No: KU18200). All procedures were performed according to approved protocols and recommendations for the proper care and use of animals at the specificpathogen-free housing facility at Konkuk University.

To produce tumor, HeyA8 cells $\left(\begin{array}{lll}1 & \mathrm{x} & 10^{6}\end{array}\right)$ were injected intraperitoneally (i.p.). The mice were grouped as follows ( $\mathrm{n}=5$ mice per group): (1) PBS as a placebo negative control, (2) LN-NEs (LN; $100 \mathrm{mg} / \mathrm{kg}$, i.v., twice a week), (3) DOX-liposomes (DOX; $5 \mathrm{mg} / \mathrm{kg}$, i.v., once a week), and (4) LN-NE-DOX-liposomes (both LN; $100 \mathrm{mg} / \mathrm{kg}$ and DOX; $5 \mathrm{mg} / \mathrm{kg}$, i.v., once a week, and $\mathrm{LN} ; 100 \mathrm{mg} / \mathrm{kg}$, i.v., 3 days after LN-NE-DOX-liposome injection) to adjust the therapeutic dose of LN. Treatment continued until the mice became moribund (typically 3 to 4 weeks). Tumor weight, number of tumor nodules, and mouse weight were recorded. The individuals who performed the necropsies, tumor collections, and tissue processing were blinded to the treatment group assignments. Tissue specimens were fixed with optimum cutting temperature (OCT, Scigen Scientific, Gardena, CA, USA) and $4 \%$ formaldehyde. 


\section{Immunohistochemistry}

Immunohistochemistry (IHC) analysis was performed by staining tumor tissues for markers of cell proliferation (anti-Ki67, Abcam, Cambridge, UK), microvessel density (anti-CD31, Abcam, Cambridge, UK), and apoptosis (anticaspase-3, Abcam, Cambridge, UK). ${ }^{23}$ Five random fields were imaged from each slide and quantified by two investigators in a blinded manner.

\section{Biochemical Toxicity of Liposomal} Formulations

Female BALB/c nude mice (5-6 weeks old, 20 g) were purchased from ORIENT (Gapyeong, South Korea). The mice were grouped as follows ( $\mathrm{n}=3$ mice per group): (1) PBS as a negative control, (2) NEs, (3) liposomes, and (4) NE-liposomes. The liposomal formulations were injected intravenously (i.v.) by a single treatment, the same as the therapeutic dose. To determine the biochemical toxicity of the liposomal formulations, the levels of aspartate aminotransferase (AST), alanine aminotransferase (ALT), and blood urea nitrogen (BUN) were analyzed using diagnostic kits (Roche, Berlin, Germany). ${ }^{27}$ Blood samples were collected from the mice through the retro-orbital sinus on days 1 and 7 after the injection of liposomes. Serum was obtained from the blood by centrifugation at $3000 \mathrm{rpm}$ at $4{ }^{\circ} \mathrm{C}$ for $10 \mathrm{~min}$ (Smart R17 Plus, Hanil Scientific Inc., Seoul, South Korea).

\section{Statistical Analysis}

Differences in continuous variables were analyzed using Student's $t$-test to compare two groups. Analysis of variance (ANOVA) was carried out to compare the differences between multiple groups. A p-value of $<0.05$ was considered statistically significant.

\section{Results}

\section{Characteristics of LN-NE-DOX-}

\section{Liposomes}

LN-NEs were prepared with the aim to overcome the insolubility of LN, as previously reported. ${ }^{17}$ We next prepared LN-NE-DOX-liposomes that encapsulated LN-NEs and DOX (Figure 1). The size of LN-NEs, liposomes, DOXliposomes, and LN-NE-DOX-liposomes was $50.6 \pm 0.2$ $\mathrm{nm}, 118.5 \pm 3.2 \mathrm{~nm}, 129.8 \pm 6.1 \mathrm{~nm}$ and $267.0 \pm 4.6 \mathrm{~nm}$, respectively, and their zeta potentials were $-31.4 \pm 1.2 \mathrm{mV}$, $-70.0 \pm 1.6 \mathrm{mV},-76.6 \pm 3.2 \mathrm{mV}$, and $-75.0 \pm 5.1 \mathrm{mV}$,
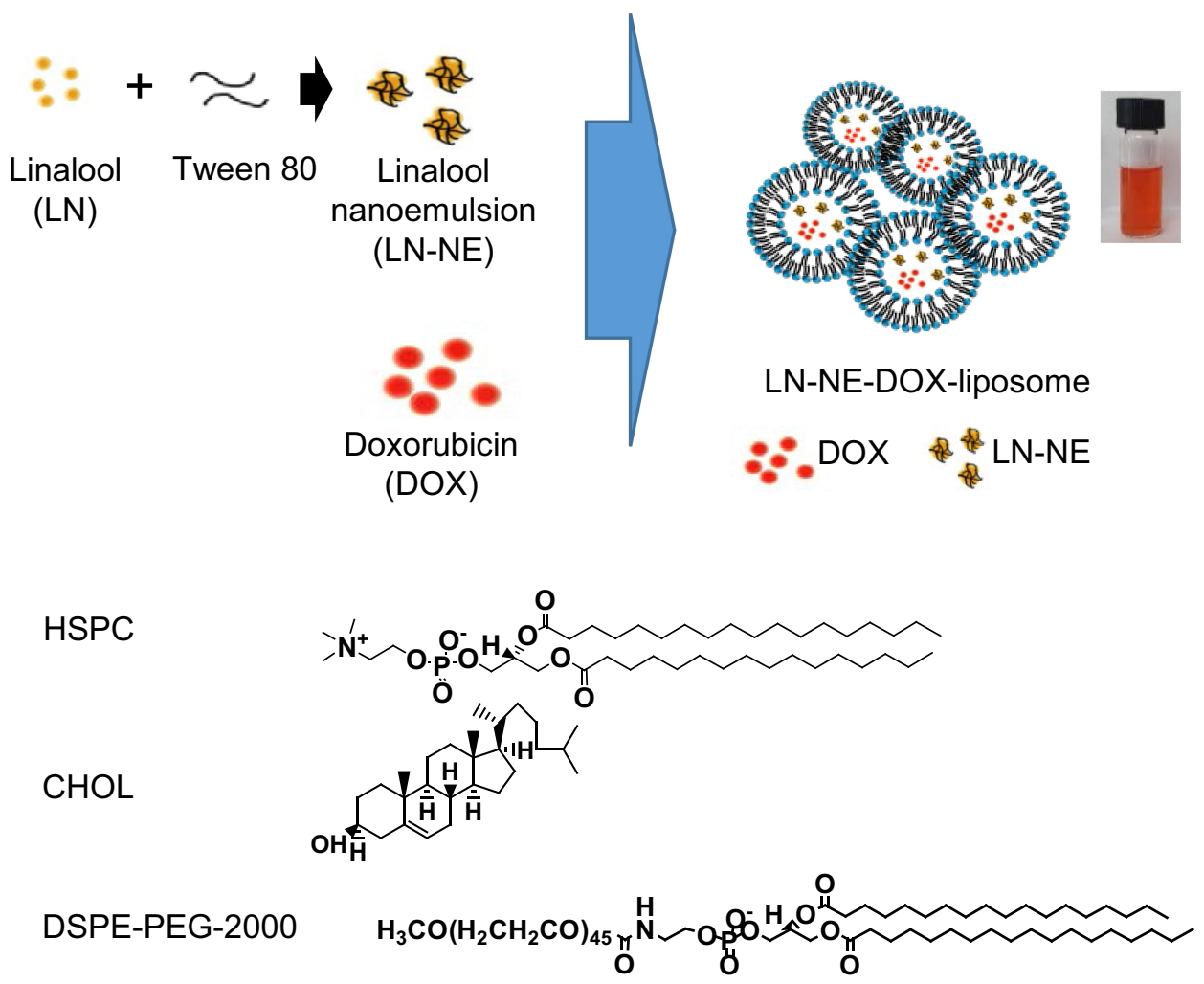

Figure I Schematic illustration of the preparation of LN-NE-DOX-liposomes. 
respectively (Figure 2A and B). The size of the LN-NE-DOX -liposomes increased slightly with decreasing zeta potential compared to those of liposomes and DOX-liposomes. A histogram of the size distribution of liposomal formulations is shown in Supplementary Figure S1 and the morphologies as observed by cryo-TEM are shown in Figure $2 \mathrm{C}$. The loading efficiency of LN into LN-NE-DOX-liposomes was $55.1 \pm 3.1 \%$, and that of DOX into DOX-liposomes and LNNE-DOX-liposomes was $80.2 \pm 2.8 \%$ and $27.2 \pm 0.9 \%$, respectively (Figure 2D and E). LN-NE-DOX-liposomes showed a relatively lower DOX loading efficiency than DOX-liposomes. As shown in Figure 2C, LN-NEs were loaded into LN-NE-DOX-liposomes in the first step, which may have resulted to the inner space of the LN-NE-DOXliposome being reduced. Therefore, LN-NE-DOX-liposomes showed lower loading efficiency than DOX-liposomes (Figure 2E). In addition, the encapsulation of LN and DOX into LN-NE-DOX-liposomes was confirmed by UV-vis spectroscopy at $295 \mathrm{~nm}$ and $490 \mathrm{~nm}$ for LN and DOX, respectively, indicating that both $\mathrm{LN}$ and DOX were completely encapsulated in the LN-NE-DOX-liposomes (Figure 2F). Moreover, we confirmed the stability of LN-NE-DOXliposomes in a $10 \%$ FBS solution and the drug release behavior from LN-NE-DOX-liposomes in PBS (Supplementary Figure S2). Liposomal formulations showed a small size change, indicating their stability in 10\% FBS (Supplementary Figure S2A). The release of LN or DOX from LN-NE-DOX-liposomes was increased with respect to time in PBS at $37^{\circ} \mathrm{C}$ (Supplementary Figure S2B).

\section{Cytotoxicity of LN-NE-DOX-Liposomes}

We next assessed the in vitro cytotoxicity of LN-NE-DOX -liposomes in HeyA8 EOC cells via the MTT assay. ${ }^{17}$ The cytotoxicity of LN-NEs increased with increasing concentration, but there was no significant difference with respect to incubation time (Figure 3A). Notably, the cytotoxicity of LN-NE-DOX-liposomes $\left(\mathrm{IC}_{50}: 0.25 \mu \mathrm{M}\right)$ significantly increased with increasing DOX concentration compared to those of DOX alone ( $\left.\mathrm{IC}_{50}: 1.18 \mu \mathrm{M}\right)$ and DOX-liposomes $\left(\mathrm{IC}_{50}: 2.81 \mu \mathrm{M}\right)$ at $24 \mathrm{~h}$ (Figure $\left.3 \mathrm{~B},{ }^{*} p<0.001\right)$. This result indicates that the combination of $\mathrm{LN}$ and DOX significantly increased cell cytotoxicity. In addition, we evaluated the apoptotic effect of the combination treatment against HeyA8 EOC cells (Figure 3C). LN-NE-DOXliposomes induced a significant increase in apoptosis at $72 \mathrm{~h}$ compared to $\mathrm{LN}-\mathrm{NE}$ and DOX-liposomes $(* p<$ 0.001). This result was in agreement with the MTT assay and indicated that the combination of $\mathrm{LN}$ and $\mathrm{DOX}$ showed a greatly increased therapeutic effect.
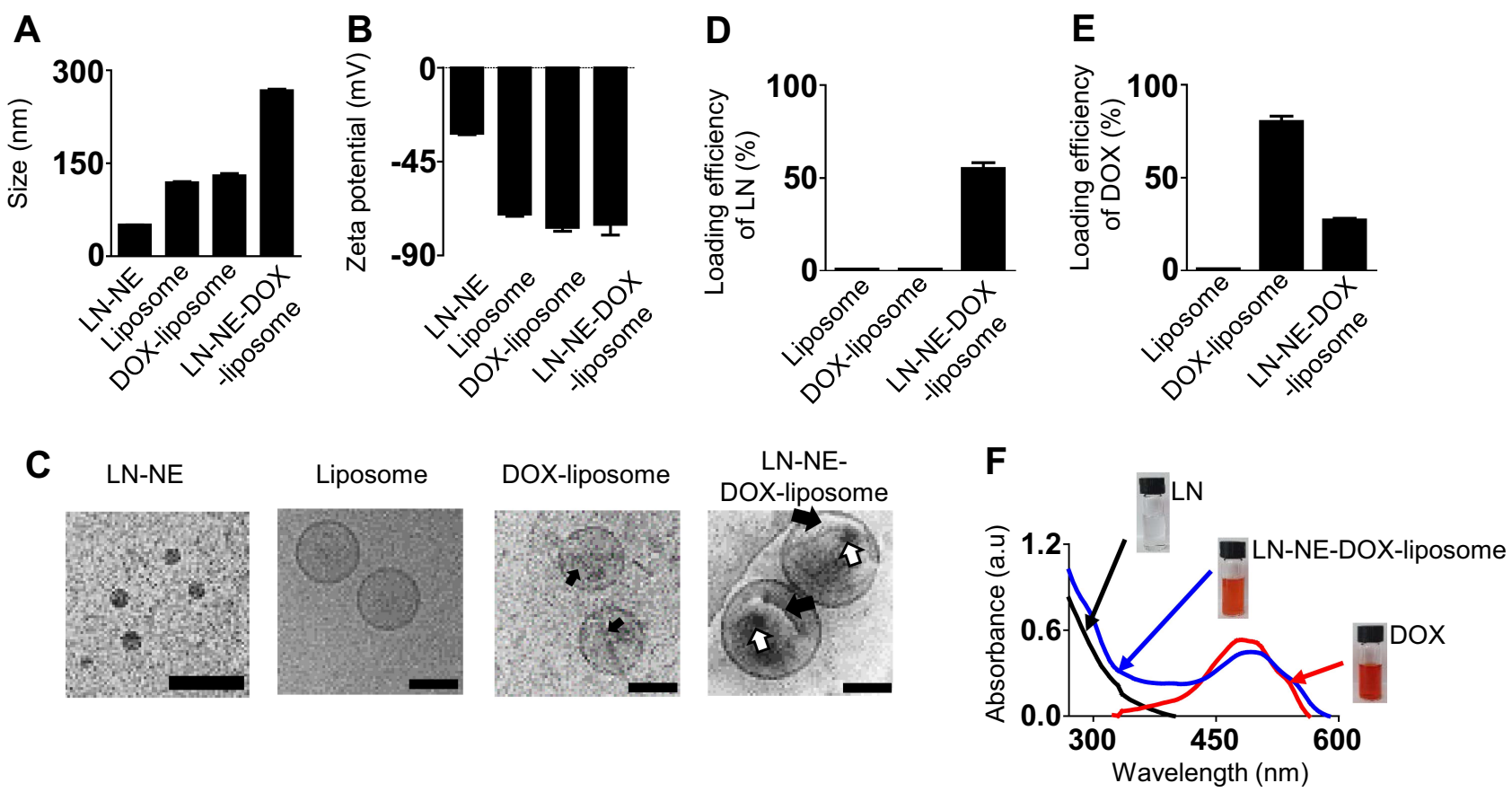

Figure 2 Physical properties of the liposomal formulations. (A) Size and (B) zeta potential of liposomes were measured by laser light scattering using a particle size analyzer. (C) Morphologies of liposomes were evaluated by cryo-TEM. The arrow denotes encapsulation of DOX and LN-NE (scale bar: I00 nm). The loading efficiency of (D) LN and (E) DOX was measured by UV-vis spectrophotometry. (F) Encapsulation of LN and DOX into LN-NE-DOX liposomes was confirmed by UV-vis spectrophotometry. The data are presented as the mean \pm S.D. $(n=3)$. 


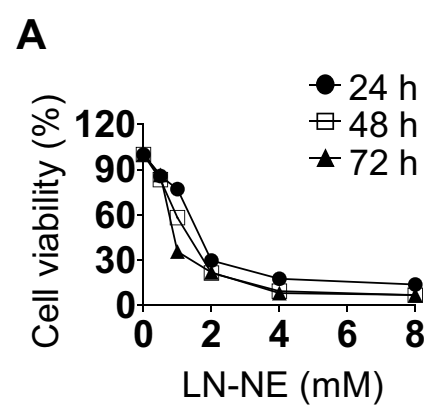

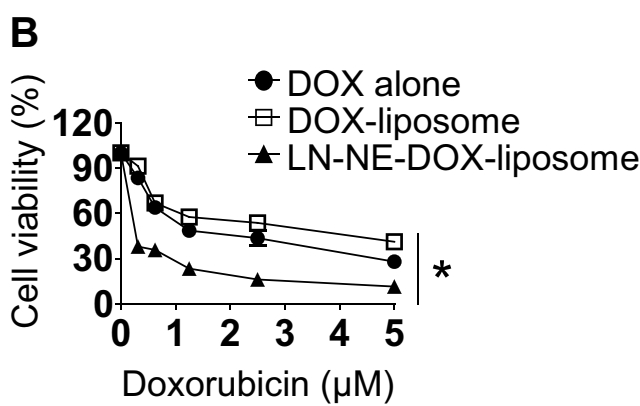
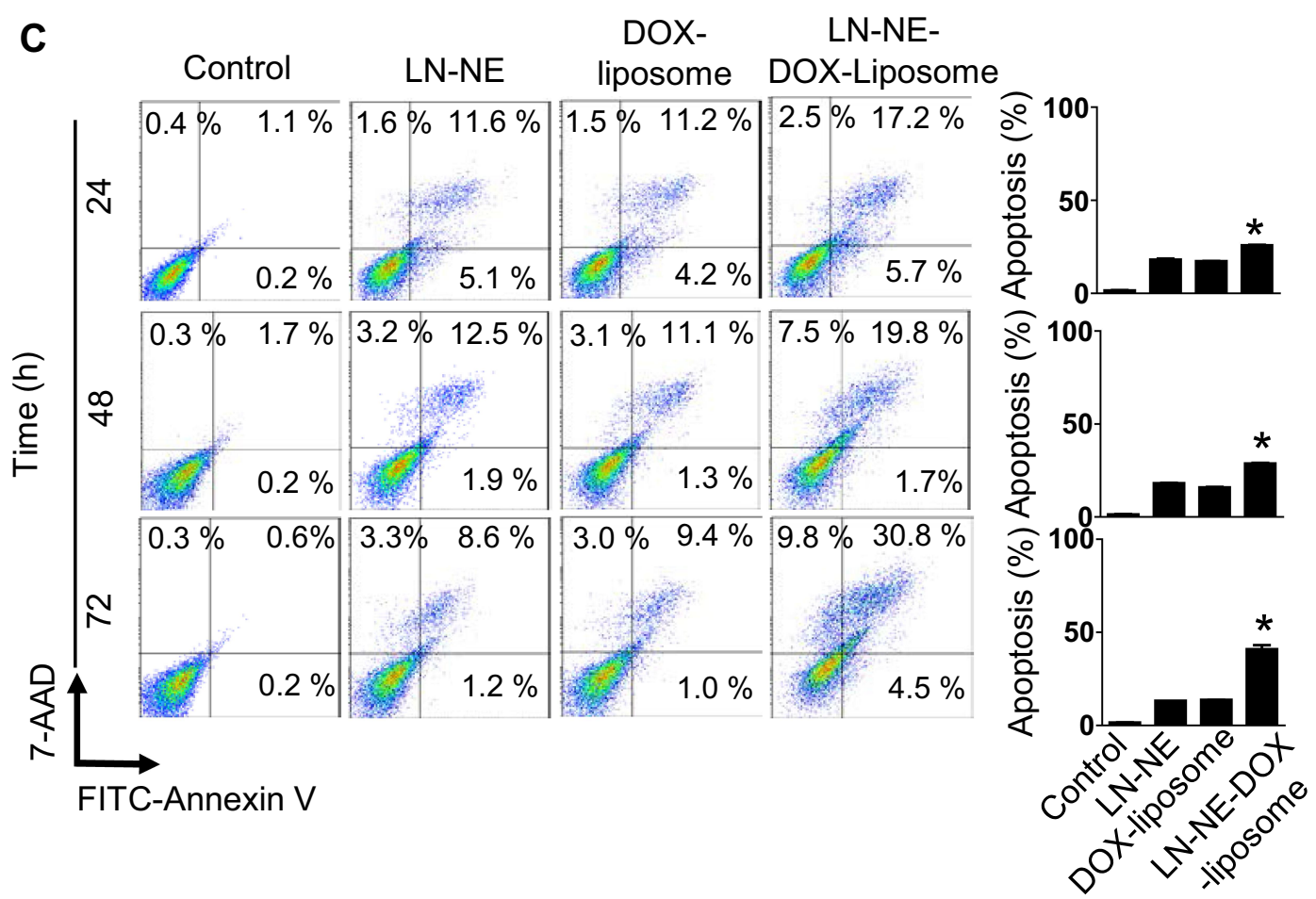

Figure 3 Cell viability and apoptosis. Cytotoxicity of $(\mathbf{A}) \mathrm{LN}-\mathrm{NE}(24 \mathrm{~h}, 48 \mathrm{~h}$, and $72 \mathrm{~h})$ and $(\mathbf{B})$ DOX against HeyA8 cells $\left({ }^{*} p<0.00 \mathrm{I}\right)$. (C) Apoptosis of HeyA8 cells cultured with LNNEs, DOX-liposomes, or LN-NE-DOX-liposomes $(* p<0.00 \mathrm{I})$. The concentration of DOX or LN used was $0.5 \mu M$ or $0.3 \mathrm{mM}$, respectively. The data are presented as the mean \pm S.D. $(n=3)$.

\section{Therapeutic Efficacy of LN-NE-DOX- Liposomes}

To confirm the therapeutic efficacy of LN-NE-DOXliposomes in the HeyA8 EOC tumor model, mice were injected with HeyA8 cells intraperitoneally. The mice were grouped as follows ( $\mathrm{n}=5$ mice per group): (1) control, (2) LN-NEs, (3) DOX-liposomes, and (4) LNNE-DOX-liposomes. LN (twice a week) and DOX (once a week) were injected intravenously at a dose of $100 \mathrm{mg} / \mathrm{kg}$ and $5 \mathrm{mg} / \mathrm{kg}$, respectively (Figure 4A). LN-NE-DOX-liposomes significantly inhibited tumor growth compared to that of the control $(75 \%$ reduction, $* * * p<0.001)$, LN-NEs $(68 \%$ reduction, $* * p<0.01)$, or
DOX-liposomes $\left(55.5 \%\right.$ reduction, $\left.{ }^{*} p<0.05\right)$ (Figure 4B). Moreover, LN-NE-DOX-liposomes significantly decreased the number of tumor nodules compared to that found in the control $(* * * p<0.01)$, LN-NE $(* * p<$ $0.03)$, or DOX-liposomes $(* p<0.05$, Figure $4 \mathrm{C})$. There was no significant difference in the mean body weight of the mice, suggesting that there was no overt therapyrelated toxicity $(* p>0.05$, Figure 4D). In addition, LN-NE-DOX-liposomes significantly reduced cell proliferation (Ki67) and microvessel density (MVD, CD31) and promoted apoptosis compared to those of the control group $(* * * p<0.001)$, LN-NEs $(* * p<0.01, * p<0.05)$, or DOX-liposomes $(* p<0.05)$ (Figure $4 \mathrm{E}$ ). 
$100 \mathrm{mg} / \mathrm{kg} \mathrm{LN}$

(twice a week)
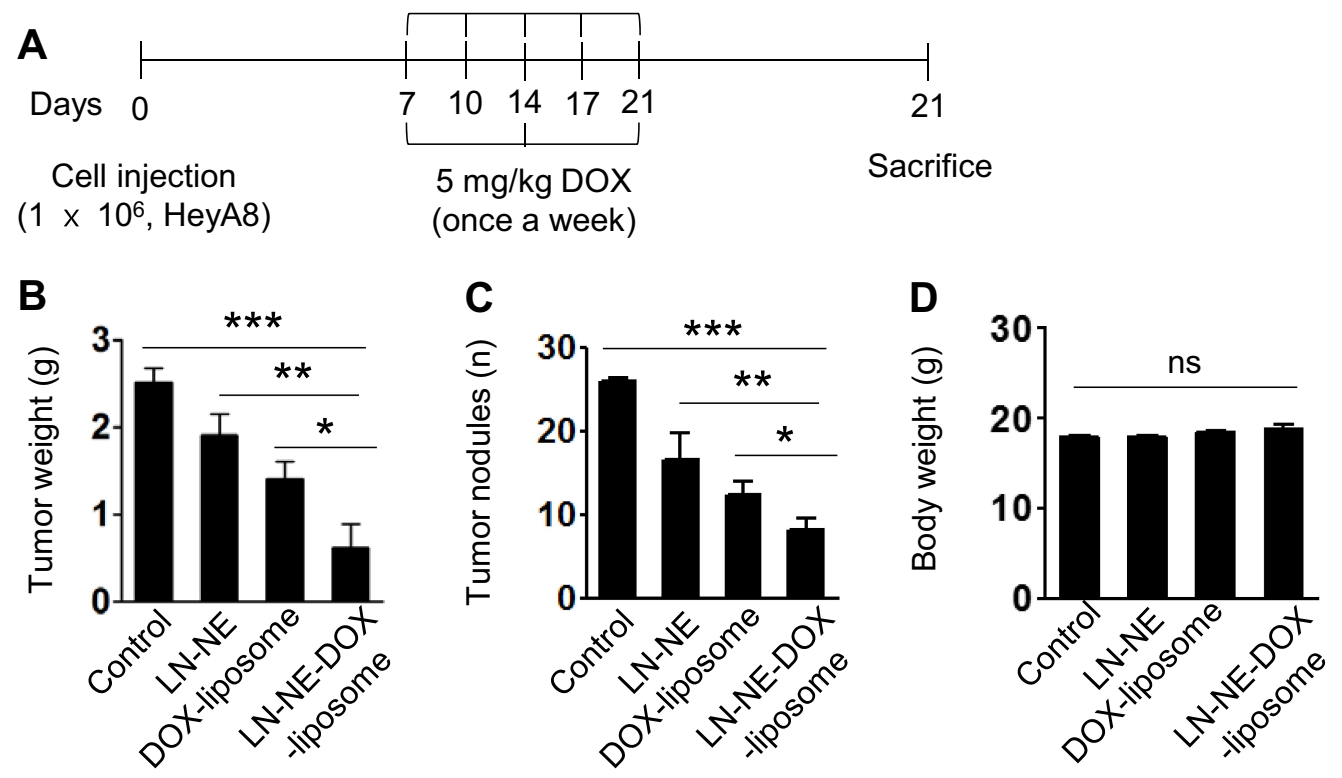

E
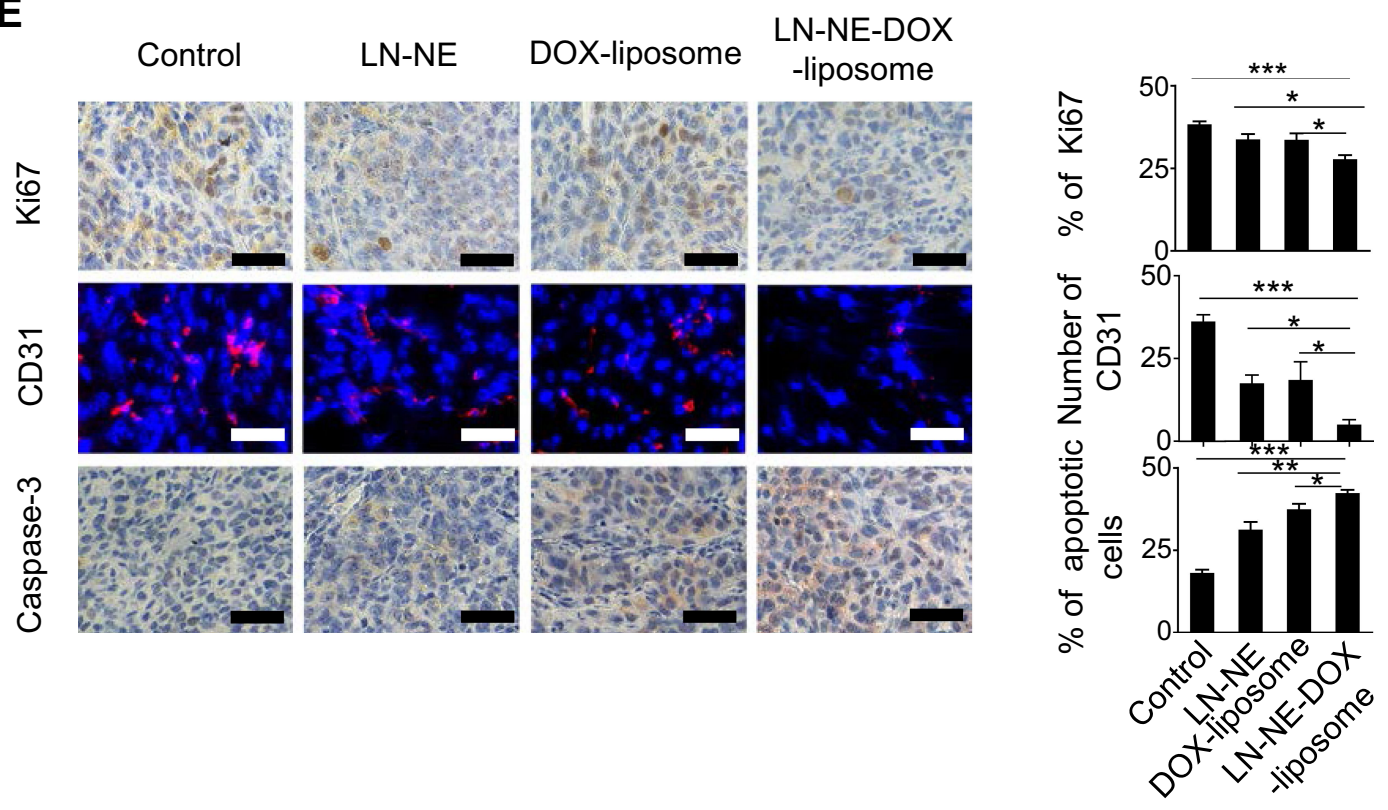

Figure 4 Therapeutic efficacy of LN-NE-DOX-liposomes in a HeyA8 tumor model. Liposomal formulations were injected intravenously at a dose of I00 mg/kg LN (twice a week) or $5 \mathrm{mg} / \mathrm{kg}$ DOX (once a week) based on body weight. (A) Experimental timeline. (B) Tumor weight, (C) tumor nodules, and (D) body weight. (E) Immunohistochemical analyses for cell proliferation (Ki67), microvessel density (CD3I), and apoptosis (caspase-3) were performed in HeyA8 tumor tissue (scale bar: $50 \mu \mathrm{m}) .(* p<0.05, * * p<0.01$, and $* * * p<0.001)$. The data are presented as the mean \pm S.D. $(\mathrm{n}=5)$.

\section{Biochemical Toxicity of Liposomal Formulations}

To evaluate the biochemical toxicity of liposomal formulations on liver function (ALT and AST) and renal function (BUN), we analyzed the levels of relevant biomarkers in the serum of mice after they were injected with these liposomal formulations. AST, ALT, and BUN levels showed no significant differences between the liposomal formulations and the control ( $p>0.05$, Figure 5A) following the guidelines for toxicity (normal range of 54-298 IU/L for AST, 17-77 IU/L for ALT, and $8-33 \mathrm{mg} / \mathrm{dL}$ for BUN). ${ }^{28}$ Moreover, H\&E staining for vital organs was also performed to evaluate liposomal formulation-induced toxicity. The histological structures of the organs treated with the 

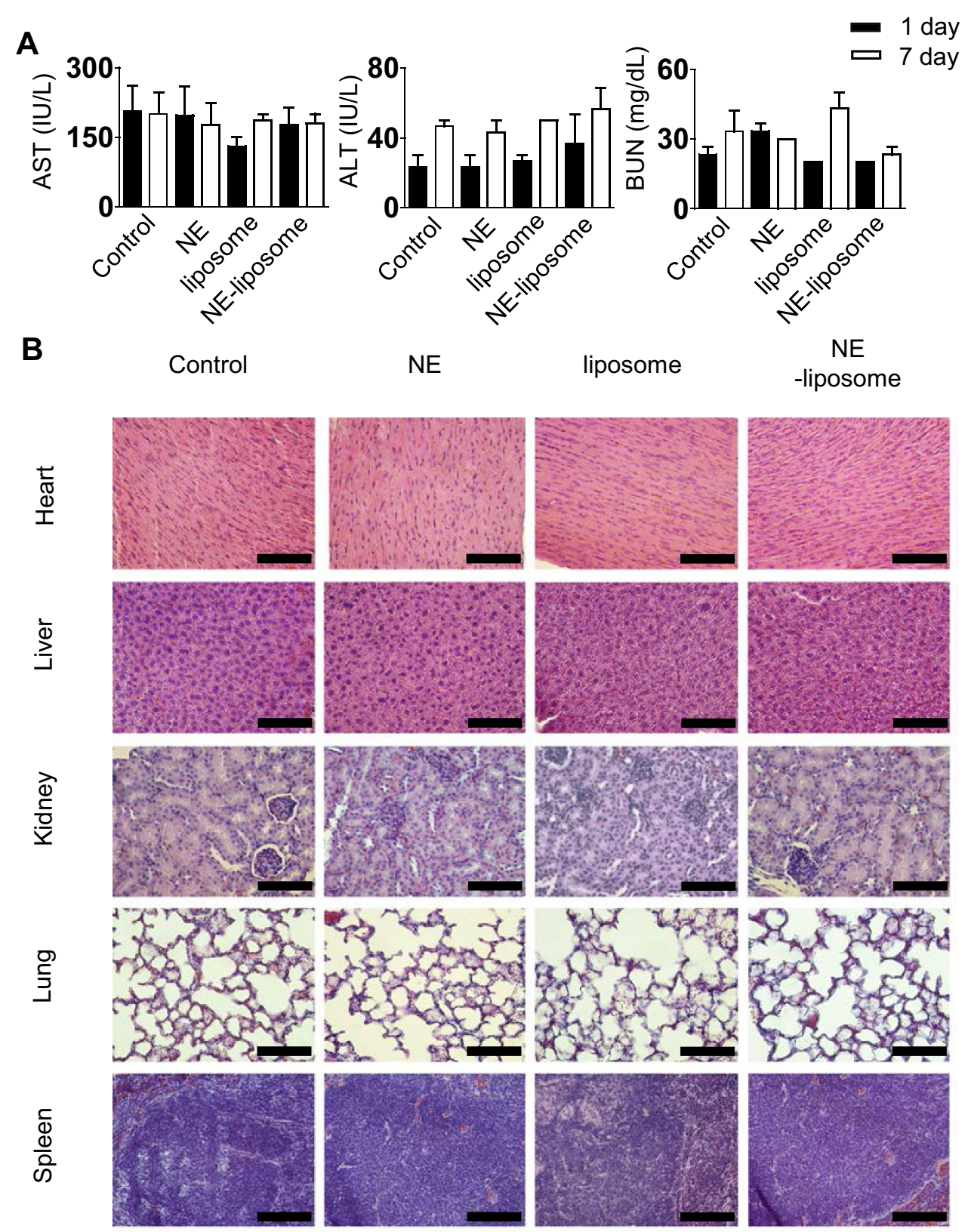

Figure 5 Biochemical toxicity of liposomal formulations. (A) Levels of the biomarkers AST, ALT, and BUN were evaluated using diagnostic kits by analyzing the serum of mice after injection of liposomes. (B) H\&E staining of major organs collected from mice (scale bar: $100 \mu m$ ). The data are presented as the mean \pm S.D. $(n=3)$.

liposomal formulations were similar with the control group, showing no differences in pathological observations (Figure 5B). These results indicate that liposomes show consistent histology comparable to that of the normal condition.

\section{Discussion}

In this study, we demonstrated the therapeutic efficacy of LN and DOX in a mouse model of EOC. We successfully developed LN-NE-DOX-liposomes as a two-in-one delivery system for both LN and DOX. This delivery system improves their anticancer effect through synergy and can reduce side effects by decreasing the amount of anticancer agents used. Consequently, the LN-NE-DOX-liposomes showed synergistic toxicity and apoptosis against EOC tumor cells. Additionally, the LN-NE-DOX-liposomes significantly inhibited tumor growth $(* * * p<0.001)$ in the EOC HeyA8 tumor model without significant side effects compared to the control.

The two-in-one delivery system proposed here simultaneously delivers hydrophobic LN and hydrophilic DOX into EOC cells. LN-NE-DOX-liposomes can overcome the 
poor water solubility of LN and reduce the side effects of DOX. Moreover, LN-NE-DOX-liposomes led to an enhanced therapeutic effect for EOC. This approach has broad applications for patient treatment using chemotherapeutics by reducing the inherent side effects of anticancer drugs.

As a standard protocol, EOC patients receive chemotherapy as a secondary treatment after tumor resection. ${ }^{3}$ However, they experience side effects caused by chemotherapeutics. Combination therapy has been proposed to reduce side effects and increase treatment efficiency compared to anticancer monotherapies, ${ }^{29}$ and studies using natural products have also been conducted in combination therapy research. ${ }^{30}$ Among these natural products, LN is a terpene alcohol that exists in various plants such as lamiaceae and lauraceae and is known to be cytotoxic against tumor cells. ${ }^{14}$ However, the bioavailability of LN is low because of its insoluble properties, thus limiting its utility as an anticancer drug. ${ }^{14}$ To overcome this problem, we previously developed an LN-NE formulation as a nanoemulsion-based delivery system. ${ }^{17}$

Liposomal delivery systems have been studied as carrier systems for a variety of drugs. These systems provide an attractive lipid-based matrix for cargo delivery and are desirable for biological applications because of their low toxicity and high biocompatibility, which are important parameters for medical and pharmaceutical applications. In particular, liposomes are known to increase the blood circulation times of drugs, and are attractive carriers that can concentrate drugs in the tumor microenvironment through the enhanced permeability and retention effect. ${ }^{31}$ Therefore, these liposomal properties are a practicable application for the systemic delivery of LN-NE-DOX.

The two-in-one delivery system focused on a highly promising strategy for systemic drug delivery to tumor cells. $^{32,33}$ In this study, we suggested a novel two-in-one delivery system to enhance the therapeutic effect of two molecules by combination. The potential of this system was optimized for biomedical applications in tumor treatment and can also be a possible one-step delivery system for chemotherapeutic agents. Hence, we suggest that our nanomedicine-based platform has great potential as a twoin-one delivery system. In addition, studies will be needed to evaluate this application to other tumor types. Nevertheless, this platform plays an important role in potential biomedical applications for effective drug delivery based on a combination therapy for cancer.

\section{Conclusion}

In summary, we developed a novel delivery system for the dual delivery of LN and DOX as a two-in-one carrier system. This liposomal platform proposes a combination therapy that could be applied in EOC treatment and provides effective strategies for cancer therapy. Indeed, the liposomal formulations significantly enhanced their antitumor efficacy compared to monotherapies in preclinical EOC models. While the LN-NE can be useful as an antitumor drug, additional approaches may be useful. Nevertheless, the combination strategy presented here has broad potential as a nanomedicine platform in human disease and could be adapted for other cancer models.

\section{Funding}

This work was supported by the National Research Foundation of Korea (NRF) funded by the Korean government (NRF-2016R1A5A2012284 and NRF2019R1A2C1085144 to Y.-M.P. and H.D.H.).

\section{Disclosure}

The authors declare that they have no competing financial or non-financial interests.

\section{References}

1. Brett MR, Jennifer B. P, Thomas A. S. Epidemiology of ovarian cancer: a review. Cancer Biol Med. 2017;14(1):9-32. doi:10.20892/ j.issn.2095-3941.2016.0084.

2. Tsao Y, Creedy DK. Auricular acupressure: reducing side effects of chemotherapy in women with ovarian cancer. Support Care Cancer. 2019;27(11):4155-4163. doi:10.1007/s00520-019-04682-8.

3. Cristea M, Han E, Salmon L, Morgan RJ. Practical considerations in ovarian cancer chemotherapy. Ther Adv Med Oncol. 2010;2 (3):175-187. doi:10.1177/1758834010361333.

4. Virani NA, Thavathiru E, McKernan P, Moore K, Benbrook DM, Harrison RG. Anti-CD73 and anti-OX40 immunotherapy coupled with a novel biocompatible enzyme prodrug system for the treatment of recurrent, metastatic ovarian cancer. Cancer Lett. 2018;425:174-182. doi:10.1016/j.canlet.2018.03.027.

5. Yahara K, Ohguri $T$, Imada $H$, et al. Epithelial ovarian cancer: definitive radiotherapy for limited recurrence after complete remission had been achieved with aggressive front-line therapy. $J$ Radiat Res. 2013;54(2):322-329. doi:10.1093/jrr/rrs108.

6. Hu J, Shen Y, Zhang G, et al. Effect of acupoint therapies on chemotherapy-induced nausea and vomiting: A systematic review protocol. Medicine. 2019;98(37):e17109. doi:10.1097/md.00000000 00017109.

7. Ruggeri C, Gioffré S, Achilli F, Colombo GI, D’Alessandra Y. Role of microRNAs in doxorubicin-induced cardiotoxicity: an overview of preclinical models and cancer patients. Heart Fail Rev. 2018;23 (1):109-122. doi:10.1007/s10741-017-9653-0.

8. Jung SH, Kim SK, Jung SH, et al. Increased stability in plasma and enhanced cellular uptake of thermally denatured albumin-coated liposomes. Colloids Surf B Biointerfaces. 2010;76(2):434-440. doi:10.1016/j.colsurfb.2009.12.002. 
9. Krauss AC, Gao X, Li L, et al. FDA approval summary: (Daunorubicin and Cytarabine) liposome for injection for the treatment of adults with high-risk acute myeloid leukemia. Clin Cancer Res. 2019;25(9):2685-2690. doi:10.1158/1078-0432.ccr-18-2990.

10. Barenholz Y. Doxil ${ }^{\circledR}$ - the first FDA-approved nano-drug: lessons learned. J Control Release. 2012;160(2):117-134. doi:10.1016/j. jconrel.2012.03.020.

11. Pistollato F, Calderon Iglesias R, Ruiz R, et al. The use of natural compounds for the targeting and chemoprevention of ovarian cancer. Cancer Lett. 2017;411:191-200. doi:10.1016/j.canlet.2017.09.050.

12. Rajesh E, Sankari LS, Malathi L, Krupaa JR. Naturally occurring products in cancer therapy. J Pharm Bioallied Sci. 2015;7(Suppl 1): S181-183. doi:10.4103/0975-7406.155895.

13. Wei X, Shamrakov D, Nudelman S, et al. Cardinal role of intraliposome doxorubicin-sulfate nanorod crystal in doxil properties and performance. ACS Omega. 2018;3(3):2508-2517. doi:10.1021/ acsomega. $7 \mathrm{~b} 01235$.

14. Iwasaki K, Zheng YW, Murata S, et al. Anticancer effect of linalool via cancer-specific hydroxyl radical generation in human colon cancer. World J Gastroenterol. 2016;22(44):9765-9774. doi:10. 3748/wjg.v22.i44.9765.

15. Pereira I, Severino P, Santos AC, Silva AM, Souto EB. Linalool bioactive properties and potential applicability in drug delivery systems. Colloids Surf B Biointerfaces. 2018;171:566-578. doi:10.1016/j.colsurfb.2018.08.001.

16. Kalepu S, Nekkanti V. Insoluble drug delivery strategies: review of recent advances and business prospects. Acta Pharm Sin B. 2015;5 (5):442-453. doi:10.1016/j.apsb.2015.07.003.

17. Han HD, Cho YJ, Cho SK, et al. Linalool-incorporated nanoparticles as a novel anticancer agent for epithelial ovarian carcinoma. Mol Cancer Ther. 2016;15(4):618-627. doi:10.1158/1535-7163.mct-150733-t.

18. Desale SS, Soni KS, Romanova S, Cohen SM, Bronich TK. Targeted delivery of platinum-taxane combination therapy in ovarian cancer. $J$ Control Release. 2015;220(Pt B):651-659. doi:10.1016/j.jconrel. 2015.09.007.

19. Bayat Mokhtari R, Homayouni TS, Baluch N, et al. Combination therapy in combating cancer. Oncotarget. 2017;8(23):38022-38043. doi:10.18632/oncotarget.16723.

20. Pritchard JR, Bruno PM, Gilbert LA, Capron KL, Lauffenburger DA, Hemann MT. Defining principles of combination drug mechanisms of action. Proc Natl Acad Sci U S A. 2013;110(2):E170-179. doi:10.1073/pnas.1210419110.

21. Kang Y, Hu W, Ivan C, et al. Role of focal adhesion kinase in regulating YB-1-mediated paclitaxel resistance in ovarian cancer. J Natl Cancer Inst. 2013;105(19):1485-1495. doi:10.1093/jnci/ djt210.

International Journal of Nanomedicine

\section{Publish your work in this journal}

The International Journal of Nanomedicine is an international, peerreviewed journal focusing on the application of nanotechnology in diagnostics, therapeutics, and drug delivery systems throughout the biomedical field. This journal is indexed on PubMed Central, MedLine, CAS, SciSearch ${ }^{\circledR}$, Current Contents ${ }^{\circledR} /$ Clinical Medicine,
22. Lu C, Han HD, Mangala LS, et al. Regulation of tumor angiogenesis by EZH2. Cancer Cell. 2010;18(2):185-197. doi:10.1016/j.ccr.2010. 06.016 .

23. Byeon Y, Lee JW, Choi WS, et al. CD44-targeted PLGA nanoparticles incorporating paclitaxel and FAK siRNA overcome chemoresistance in epithelial ovarian cancer. Cancer Res. 2018;78(21):6247. doi:10.1158/0008-5472.can-17-3871.

24. Kwon HJ, Byeon Y, Jeon HN, Cho SH, Han HD, Shin BC. Gold cluster-labeled thermosensitive liposmes enhance triggered drug release in the tumor microenvironment by a photothermal effect. J Control Release. 2015;216:132-139. doi:10.1016/j.jconrel.2015. 08.002 .

25. Han HD, Jeon YW, Kwon HJ, et al. Therapeutic efficacy of doxorubicin delivery by a $\mathrm{CO} 2$ generating liposomal platform in breast carcinoma. Acta Biomater. 2015;24:279-285. doi:10.1016/j. actbio.2015.06.019.

26. Won JE, Byeon Y, Wi TI, et al. Enhanced antitumor immunity using a tumor cell lysate-encapsulated $\mathrm{CO} 2$-generating liposomal carrier system and photothermal irradiation. ACS Appl Bio Mater. 2019;2 (6):2481-2489. doi:10.1021/acsabm.9b00183.

27. Ou Y-C, Wen X, Johnson CA, et al. Multimodal multiplexed immunoimaging with nanostars to detect multiple immunomarkers and monitor response to immunotherapies. ACS Nano. 2020;14 (1):651-663. doi:10.1021/acsnano.9b07326.

28. Mayer LP, Dyer CA, Eastgard RL, Hoyer PB, Banka CL. Atherosclerotic lesion development in a novel ovary-intact mouse model of perimenopause. Arterioscler Thromb Vasc Biol. 2005;25 (9):1910-1916. doi:10.1161/01.ATV.0000175767.46520.6a.

29. Cortez AJ, Tudrej P, Kujawa KA, Lisowska KM. Advances in ovarian cancer therapy. Cancer Chemother Pharmacol. 2018;81 (1):17-38. doi:10.1007/s00280-017-3501-8.

30. Chamberlin SR, Blucher A, Wu G, et al. Natural product target network reveals potential for cancer combination therapies. Front Pharmacol. 2019;10:557. doi:10.3389/fphar.2019.00557.

31. Golombek SK, May J-N, Theek B, et al. Tumor targeting via EPR: strategies to enhance patient responses. Adv Drug Deliv Rev. 2018;130:17-38. doi:10.1016/j.addr.2018.07.007.

32. Sun R, Liu Y, Li S-Y, et al. Co-delivery of all-trans-retinoic acid and doxorubicin for cancer therapy with synergistic inhibition of cancer stem cells. Biomaterials. 2015;37:405-414. doi:10.1016/j. biomaterials.2014.10.018.

33. Barui S, Saha S, Mondal G, Haseena S, Chaudhuri A. Simultaneous delivery of doxorubicin and curcumin encapsulated in liposomes of pegylated RGDK-lipopeptide to tumor vasculature. Biomaterials. 2014;35(5):1643-1656. doi:10.1016/j.biomaterials.2013.10.074.
Journal Citation Reports/Science Edition, EMBase, Scopus and the Elsevier Bibliographic databases. The manuscript management system is completely online and includes a very quick and fair peer-review system, which is all easy to use. Visit http://www.dovepress.com/ testimonials.php to read real quotes from published authors. 